Acta Theriologica 45 (2): 145-153, 2000.

PL ISSN 0001-7051

\title{
A new four-parameter, generalized logistic equation and its applications to mammalian somatic growth
}

\author{
Xinrong WAN, Mengjun WANG, Guanghe WANG and Wenqin ZHONG
}

Wan X., Wang M., Wang G. and Zhong W. 2000. A new four-parameter, generalized logistic equation and its applications to mammalian somatic growth. Acta Theriologica 45: $145-153$.

A new mathematical equation is introduced in this paper:

$$
W=f-\frac{1}{\frac{b}{f}+\left(\frac{1}{f-s}-\frac{b}{f}\right) \exp (k t)}
$$

where $W$ is the size at any convenient unit of time $t, s$ is the initial size, $f$ is the upper asympotic size, $k$ is the growth coefficient $(k>0)$, and $b$ is the constant. The new equation encompasses the logistic equation and therefore should be considered as a generalized version of the classical logistic equation. With its additional fourth parameter $b$, the new equation yields an unfixed value of inflexion point which enables it to possess good flexibility for depicting diverse growth patterns. In order to evaluate the fitness of the new growth equation, some commonly encountered models are compared to the new one using 12 sets of somatic growth data of mammalian species including hamster, rat, vole, pika, mouse, rabbit, cattle, and bear. The new equation possesses excellent fitness to each data set, suggesting that it is worth being considered by growth data analysts.

Institute of Zoology, Chinese Academy of Sciences, Beijing 100080, China, e-mail: xwan@public.east.cn.net (XW, MW, GW, WZ); The Chinese State Key Lab of Integrated Management of Pest Insects \& Rodents (XW, MW, WZ)

Key words: somatic growth, growth model, logistic equation, mammals

\section{Introduction}

Many growth equations have been used for many years in the life sciences to provide a mathematical summary of time-course data on growth of organisms, organs or tissues (Parks 1982, Banks 1994, France et al. 1996, Bazjer et al. 1997). The logistic function (Verhulst 1838, Robertson 1908) is one of the most classic growth models. Its major advantages are mathematical simplicity and apparent biological interpretation. It has played a central role in many aspects of theoretical and applied ecology (Krebs 1996).

Although the logistic equation has been extensively used in a wide range of ecological situations, its theoretical assumptions are too simple and open to much criticism (Maynard Smith 1974, Cui and Lawson 1982a). In reality, the relationship between the relative growth rate $d W /(W d t)$ and size $W$ is not necessarily linear (Cui 
Table 1. The expressions of some commonly applied growth models. Parameters $s$ and $f$ denote the initial and the upper asympotic size respectively, $k$ is the growth coefficient. Other parameters may have different biological interpretations.

\begin{tabular}{lll}
\hline Model & \multicolumn{1}{c}{ Expression } & \multicolumn{1}{c}{ References } \\
\hline Spillman & $W=f-(f-s) \exp (-k t)$ & Spillman and Lang 1924 \\
Gompertz & $W=f \exp (\ln (s / f) \cdot \exp (-k t))$ & Gompertz 1825 \\
Bertalanffy & $W=\left(f^{1 / 3}-\left(f^{1 / 3}-s^{1 / 3}\right) \cdot \exp (-k t)\right)^{3}$ & Bertalanffy 1957, Gille and Salomon 1995 \\
Logistic & $W=s \cdot f /\{s+(f-s) \exp (-k t)\}$ & Verhulst 1838, Robertson 1908 \\
Richards & $W=s \cdot f /\left\{s^{n}+\left(f^{n}-s^{n}\right) \exp (k t)\right\}^{1 / n}$ & Richards 1959 \\
Janoschek & $W=f-(f-s) \cdot \exp \left(-k t^{p}\right)$ & Gille and Salomon 1995 \\
\hline
\end{tabular}

and Lawson 1982a, b). For this reason, the logistic model fails to fit somatic growth data satisfactorily in some cases (Ricklefs 1968, Cui and Lawson 1982a, Jolicoeur et al. 1992a, b, Wan et al. 1998b). In order to enable it to fit data sets better, many attempts have been made to construct a generalized form of ordinary 3-parameter logistic equation by various authors (Richards 1959, Cui and Lawson 1982a, Jolicoeur and Pontier 1989, Jolicoeur et al. 1992b, Wan et al. 1998a).

The present paper considers a new generalized form of the ordinary logistic equation. In order to evaluate the appropriateness of this new equation, we used several commonly applied growth models, viz: the logistic, Gompertz, Bertalanffy, Spillman, Richards, Janoschek, and the present one to fit 12 sets of somatic growth data of mammalian species. The expressions of these growth models are presented in Table 1.

\section{Mathematical properties of the new model}

The new model gives:

$$
W=f-\frac{1}{\frac{b}{f}+\left(\frac{1}{f-s}-\frac{b}{f}\right) \exp (k t)}
$$

Substituting $t=0$ in equation (1) yields $W=s$, and setting $t \rightarrow \infty$ gets $W=f$. Thus, parameters $s$ and $f$ are easily to define as birth size and mature size, respectively. Parameter $k$ is the so-called growth coefficient. The first and second derivative functions can be obtained by differentiate equation (1) with respect to time $t$. The results are:

$$
\begin{gathered}
\frac{d W}{d t}=\frac{k\left(\frac{1}{f-s}-\frac{b}{f}\right) \exp (k t)}{\left[\frac{b}{f}+\left(\frac{1}{f-s}-\frac{b}{f}\right) \exp (k t)\right]^{2}} \\
\frac{d^{2} W}{d t^{2}}=\frac{k^{2} \exp (k t)\left(\frac{1}{f-s}-\frac{b}{f}\right)\left[\frac{b}{f}-\left(\frac{1}{f-s}-\frac{b}{f}\right) \exp (k t)\right]}{\left[\frac{b}{f}+\left(\frac{1}{f-s}-\frac{b}{f}\right) \exp (k t)\right]^{3}}
\end{gathered}
$$


Setting the second derivative $\frac{d^{2} W}{d t^{2}}$ equals to zero yields the POI (point of inflexion) of time $t^{\prime}$ :

$$
t^{\prime}=\frac{1}{k}\left[\ln \left(\frac{b}{f}\right)-\ln \left(\frac{1}{f-s}-\frac{b}{f}\right)\right]
$$

Substituting $t=t^{\prime}$ into equation (1) yields the POI of size $W^{\prime}$ :

$$
W^{\prime}=f\left(1-\frac{1}{2 b}\right)
$$

Unlike the ordinary 3-parameter logistic equation which has a fixed value of POI located at $f / 2$, this new model possesses a unfixed value of POI according to its additional fourth parameter $b$. To illustrate, as $b<\frac{f}{2(f-s)}$, equation (5) gives $W^{\prime}<s$ or $W^{\prime}>f$, therefore the new equation has no POI; as $b \geq \frac{f}{2(f-s)}$, equation

(5) can give any value of POI ranging from $s$ to $f$ according to a certain value of parameter $b$. For this reason, the new function has good flexibility in portraying diverse growth courses.

In addition, at the point of inflexion, the degree of maturation gives:

$$
\frac{W^{\prime}}{f}=\frac{f(1-1 / 2 b)}{f}=1-\frac{1}{2 b}
$$

Thus, parameter $b$ is a shape parameter, controlling the shape of the growth curve and adjusting the degree of maturation when growth rate peaks.

Returning to the analysis, the inverse function of equation (1) gives:

$$
t=\frac{1}{k}\left[\ln \left(\frac{1}{f-W}-\frac{b}{f}\right)-\ln \left(\frac{1}{f-s}-\frac{b}{W_{f}}\right)\right]
$$

Substituting equation (7) into equation (2) yields:

$$
\frac{d W}{d t}=-\frac{k b}{f} W^{2}+(2 b-1) k W+(1-b) k f
$$

From equation (8) we can conclude that the first derivative of the new model is essentially a general parabolic function. In a special case as $b=1$, equation (8) reduces to:

$$
\frac{d W}{d t}=-\frac{k}{f} W^{2}+k W=k W\left(\frac{f-W}{f}\right)
$$

Clearly, the new model is essentially a four-parameter, generalized logistic equation. The feature of the first derivative of this new model with respect to various values of parameter $b$ are shown in Fig. 1, indicating that the new model encompasses the classical logistic equation. In addition, we can easily get the integral form of the ordinary logistic expression by substituting $b=1$ into equation (1). This result is:

$$
W=s f /[s+(f-s) \exp (-k t)]
$$




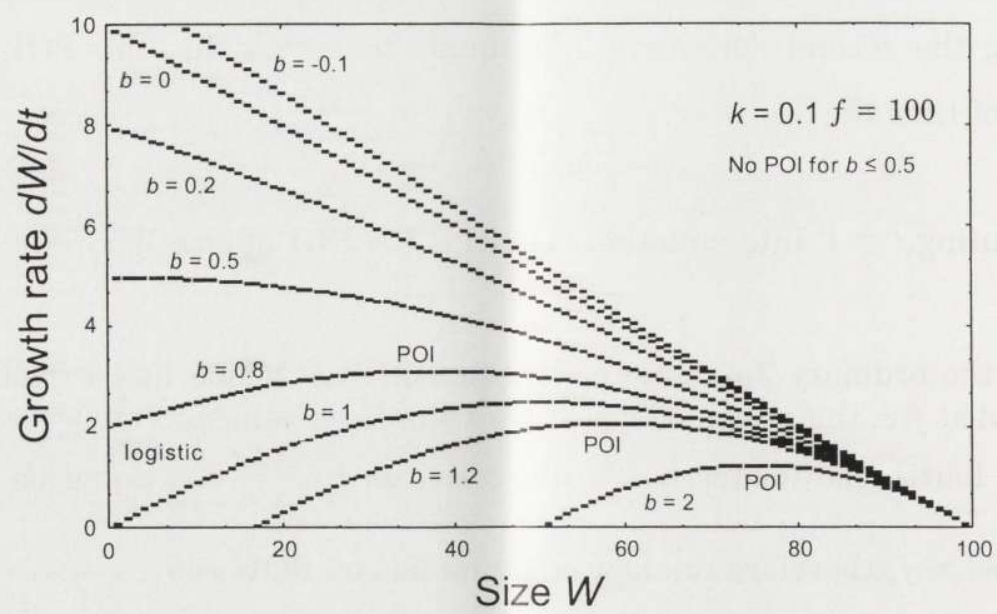

Fig. 1. The feature of the first derivative of the new model with respect to a various values of parameter $b$. As $b<f /[2(f-s)]$, there is no POI; as $b \geq f /[2(f-s)]>0.5$, a sigmoidal curve will be observed. The new model possesses unfixed value of POI according to its additional fourth parameter $b$.

\section{The inverse measure of growth rate of the new model}

The inverse measure of growth rate, namely $t_{10-90}$, which represents the time required for growth between 10 to $90 \%$ of the asymptote (Ricklefs 1968), can be derived as follows. Substituting $W=0.1 f$ and $W=0.9 f$ into equation (7) yields:

$$
\begin{aligned}
& t_{10}=\frac{1}{k}\left[\ln \left(\frac{10 / 9}{f}-\frac{b}{f}\right)-\ln \left(\frac{1}{f-s}-\frac{b}{f}\right)\right] \\
& t_{90}=\frac{1}{k}\left[\ln \left(\frac{10}{f}-\frac{b}{f}\right)-\ln \left(\frac{1}{f-s}-\frac{b}{f}\right)\right]
\end{aligned}
$$

Minus $t_{90}$ by $t_{10}$ obtains the expression of $t_{10-90}$ :

$$
t_{10-90}=\frac{1}{k} \ln \left(\frac{10-b}{10 / 9-b}\right)=\frac{1}{k} \ln \left(1+\frac{80}{10-9 b}\right)
$$

In a special case as $b=1$, equation (13) reduces to the logistic $t_{10-90}$. Therefore, the above expression becomes $t_{10-90}=\frac{1}{k} \ln 81 \approx \frac{4.4}{k}$, which coincides with Ricklefs (1976).

\section{Difference between the new model and the Richards equation}

Among the earlier literature, one of the most famous 4-parameter generalized logistic equations is the Richards equation (Table 1). Its first derivative function (Richards 1959, McCallum and Dixon 1990) gives: 


$$
\frac{d W}{d t}=l W-m W^{n}
$$

where $l, m, n$ are constants. In a special case as $n=2$, equation (14) reduces to the ordinary logistic model. Nevertheless, comparing equation (8) with equation (14), we easily see that the Richards equation and the new one are quite different in their mathematical foundations.

\section{Evaluation of growth model fits with observations}

In order to compare the fits of this new equation with commonly applied ones, we used 12 sets of observations in data analysis. Data sets refer to the following measurements: Nos 1-2 - body mass of male and female plateau pika, respectively (Zhou et al. 1987); No. 3 - body mass of the Brandt vole (Wan et al. 1998a); No. 4 body mass of mouse (Koops 1986); No. 5 - body mass of rabbit (Koops 1986); Nos 6-8 - growth data (body mass, brain mass, body length) of white rat (Jolicoeur and Pirlot 1988); Nos 9-10 - body mass and body length of Fujian cattle (Liang et al. 1995); No. 11 - body length of brown bear (Zhu and Xia 1991); No. 12 - body mass growth data of 27 male striped hamsters Cricetulus barabensis, measured on repeated occasions (this study).

All of models were used to fit each data set. All of the parameters were obtained through the least squares method (Jolicoeur 1999). The values of $R^{2}$ (the percent variation accounted for, indicating the proportion of the variation explained) and RSS (residual sum of squares) were used to evaluate the fit of each model. The statistical program STATISTICA Release 4.5A (1993) was used in parameter simulating procedures. The results are presented in Tables 2-3 and Fig. 2.

Table 2. Estimated parameter values $(k, s, f, b)$, POI of time $t^{\prime}$, POI of size $W^{\prime}$, residual sum of squares (RSS), and percent of variation accounted for $\left(R^{2}\right)$ obtained by fitting the new growth model to each data set.

\begin{tabular}{lcrrrrrrr}
\hline Data set & $k$ & $s$ & \multicolumn{1}{c}{$f$} & $b$ & \multicolumn{1}{c}{$t^{\prime}$} & $W^{\prime}$ & RSS & $R^{2}$ \\
\hline No. 1 & 0.1074 & 7.88 & 122.50 & 0.9917 & 23.79 & 60.70 & 2436.00 & 0.9816 \\
No. 2 & 0.1083 & 8.80 & 122.00 & 1.0080 & 24.67 & 61.50 & 1270.80 & 0.9902 \\
No. 3 & 0.0336 & 1.59 & 38.69 & 0.6436 & 14.21 & 8.63 & 886.40 & 0.9683 \\
No. 4 & 0.1101 & 2.77 & 26.09 & 1.0580 & 25.66 & 13.80 & 8.83 & 0.9930 \\
No. 5 & 0.0190 & 31.65 & 1385.00 & 0.7122 & 44.06 & 413.00 & 1554.00 & 0.9994 \\
No. 6 & 0.0419 & 2.17 & 305.00 & 0.9016 & 51.19 & 135.90 & 3966.00 & 0.9892 \\
No. 7 & 0.0040 & 286.00 & 1891.00 & -21.5600 & - & - & 292265.00 & 0.9630 \\
No. 8 & 0.0263 & 51.90 & 241.00 & 0.4950 & - & - & 1575.00 & 0.9879 \\
No. 9 & 0.0275 & 14.12 & 369.50 & 0.8457 & 53.53 & 151.00 & 437.00 & 0.9951 \\
No. 10 & 0.0204 & 52.73 & 147.40 & 0.3564 & - & - & 23.63 & 0.9963 \\
No. 11 & 0.0468 & 218.50 & 532.20 & 1.5400 & 48.85 & 359.00 & 4420.30 & 0.9961 \\
No. 12 & 0.0444 & 1.16 & 19.10 & 0.5682 & 3.03 & 2.29 & 4.51 & 0.9964 \\
\hline
\end{tabular}


Table 3. The values of RSS obtained by fitting each model to 12 sets of growth data. We ranked the model with best fit as (1), the model with worse fit as (7) and soon for each data set. The second line of the current table is the total rank values for all of the data sets.

\begin{tabular}{|c|c|c|c|c|c|c|c|c|c|c|c|c|c|c|}
\hline \multirow{2}{*}{$\begin{array}{l}\text { Data } \\
\text { set }\end{array}$} & \multirow{2}{*}{$\begin{array}{l}\text { Logistic } \\
\text { rank: }\end{array}$} & \multirow{2}{*}{68} & \multirow[t]{2}{*}{ Gompertz } & \multicolumn{3}{|c|}{ Bertalanffy } & \multirow{2}{*}{ Spillman } & \multicolumn{3}{|c|}{ Richards } & \multicolumn{2}{|l|}{ Janoschek } & \multicolumn{2}{|l|}{ New one } \\
\hline & & & & 60 & & 56 & & 71 & & 19 & & 33 & & 29 \\
\hline o. 1 & 0 & (3) & 0 & (5) & 303 & (6) & 00 & (7) & 238 & (1) & .00 & (4) & 00 & (2) \\
\hline fo. 2 & 2.00 & (3) & 1651.00 & (5) & 2146.00 & (6) & 00 & (7) & 1267.00 & (1) & 9.00 & (4) & .00 & (2) \\
\hline fo. 3 & .00 & (7) & 70 & (5) & 00 & (3) & 20 & (6) & 67.20 & (1) & .50 & (2) & 40 & (4) \\
\hline o. 4 & 8 & (3) & & (5) & 55 & (6) & 35 & (7) & 7.902 & 2(1) & 71 & (4) & 33 & (2) \\
\hline To. 5 & 19019.00 & (7) & 5410.00 & (5) & 2692.00 & (4) & 7754.00 & (6) & 2147.00 & (3) & 2116.00 & (2) & .00 & (1) \\
\hline Io. 6 & & (6) & & (2) & & (3) & & (7) & & (1) & 00 & (4) & & (5) \\
\hline No. 7 & 672694.00 & (7) 5 & 537953.00 & (6) 4 & 90388.00 & (5) 4 & 401056.00 & & 289369.00 & & 87512.00 & (1) 29 & 5.00 & (3) \\
\hline No. 8 & 0 & (7) & 29.00 & (5) & 6.00 & (4) & 00 & (6) & .00 & (1) & 00 & (2) & & (3) \\
\hline No. 9 & 5247.00 & (7) & 2886.00 & (6) & 2119.00 & (5) & 1025.00 & (4) & 9.70 & (2) & 60.90 & (3) & 37.00 & (1) \\
\hline 10 & 0 & (7) & 0 & (6) & 6.60 & (5) & 6.90 & (4) & 37.31 & (2) & 40.90 & (3) & 23.63 & (1) \\
\hline No. 11 & 1221.00 & (4) & 1584.00 & (5) & 1728.00 & (6) & 44.00 & (7) & 604.40 & (3) & 455.6 .0 & (2) & 420.30 & (1) \\
\hline To. 12 & 19.68 & (7) & 5.07 & (5) & 3.55 & (3) & 6.84 & (6) & 3.38 & (1) & 3.51 & (2) & 4.51 & (4) \\
\hline
\end{tabular}

\section{Discussion}

In the logistic hypothesis, the maximum velocity occurs at $f / 2$, which is not necessarily the truth. A lot of authors have revealed that in many species the peak growth rate does not occur at exactly half equilibrium (Ricklefs 1968, Cui and Lawson 1982a, Wan et al. 1998b). In these cases, the ordinary logistic equation may fail to give a suitable fit to the relevant data set. One the contrary, with its additional fourth parameter $b$, the new generalization possesses an unfixed value of POI located at the point $W^{\prime}=f\left(1-\frac{1}{2 b}\right)$, which enables it to possess more flexibility for describing diverse growth patterns than the ordinary logistic equation.

The values of RSS are used to evaluate the fit of these models. The smaller a RSS value is, the better a model fit to a data set. In many cases, adding more parameters to a model always improves the goodness of fit. Therefore 4-parameter models usually yield better fits than 3-parameter ones (Gille and Salomon 1995, Wan et al. 1998a). From Table 3 we can see that the Richards, Janoschek and the new model usually yield lower values of RSS and higher values of $R^{2}$ than the logistic, Spillman, Bertalanffy, and Gompertz equations.

Based on the numerical results presented in Table 3, the new model yields the least RSS for rabbit body mass (No. 5), cattle body mass (No. 9), cattle body length (No. 10), and brown bear body length (No. 11). The new model yields better fits than the logistic, Gompertz, Bertalanffy, Spillman, Richards, and Janoschek models in $12,11,9,12,4$, and 7 out of 12 cases, respectively. According to the total 

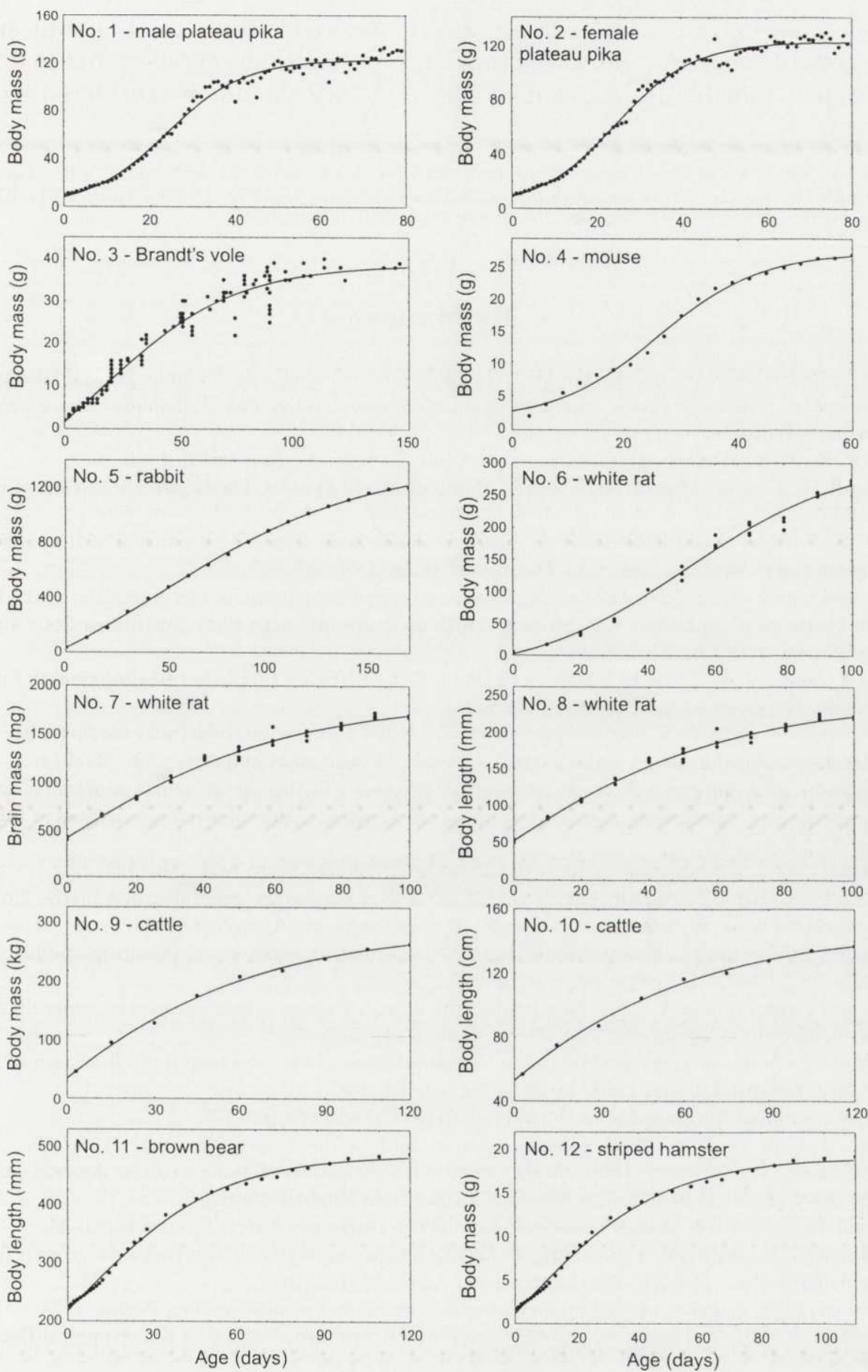

Fig. 2. Growth curves obtained by fitting the new model to 12 sets of mammalian growth data. 
of rank values given in Table 3, the new model yields the second best fit among these growth functions, indicating that the new model has excellent fits to diverse mammalian somatic growth courses and therefore should be considered by data analysts.

Acknowledgements: We thank anonymous reviewers for their criticisms and suggestions. This work was supported by the Chinese Academy of Sciences Grants (KZ951-B1-106, KZ952-S1-107) and Chinese National Sciences Grants (96-005-01-06, 96-016-01-06, 39730090).

\section{References}

Bajzer Z., Vuk-Pavlovic S. and Huzak M. 1997. Mathematical modeling of tumor growth kinetics. [In: A survey of models for tumor-immune system dynamics. J. Adam and N. Bellomo, eds]. Birkhauser, Boston: 89-133.

Banks R. B. 1994. Growth and diffusion phenomena. Springer-Verlag, Berlin: 1-26.

Bertalanffy L. V. 1957. Quantitative laws in metabolism and growth. The Quarterly Review of Biology 32: $217-231$.

Cui Q. and Lawson G. J. 1982a. Study on models of single populations: an expansion of the logistic and exponential equations. Journal of Theoretical Biology 98: 645-659.

Cui Q. and Lawson G. J. 1982b. A new model of single-species population - by extending of the logistic and exponential equations with several limiting conditions. Acta Ecologica Sinica 2(4): 403-415. [In Chinese with English summary]

France J., Dijkstra J., Thornley J. H. M. and Dhanoa M. S. 1996. A simple but flexible growth function. Growth, Development and Aging 60: 71-83.

Gille U. and Salomon F. V. 1995. Bone growth in ducks through mathematical models with special reference to the Janoschek growth curve. Growth, Development and Aging 59: 207-214.

Gompertz B. 1825. On the nature of the function expressive of the law of human mortality, and on a new method of determining the value of life contingencies. Philosophical Transactions of the Royal Society 27: 513-585.

Jolicoeur P. 1999. Introduction to biometry. Plenum Publishers, New York: 345-387.

Jolicoeur P., Cabana T. and Ducharme G. 1992a. A four-parameter generalization of the Gompertz curve suitable for somatic growth. Growth, Development and Aging 56: 69-74.

Jolicoeur P. and Pirlot P. 1988. Asymptotic growth and complex allometry of the brain and body in the white rat. Growth, Development and Aging 52: 3-10.

Jolicoeur P. and Pontier J. 1989. Population growth and decline: a four-parameter generalization of the logistic curve. Journal of Theoretical Biology 141: 563-571.

Jolicoeur P., Pontier J. and Abidi H. 1992b. Asymptotic models for the longitudinal growth of human stature. American Journal of Human Biology 4: 461-468.

Koops W. J. 1986. Multiphasic growth curve analysis. Growth 50: 169-177.

Krebs C. J. 1996. Ecology (fourth edition). Harper and Row Publishers, New York: 198-229.

Liang X., Zou X. and Xu X. 1995. Study on the growth models of Fujian cattle. Journal of Fujian Agricultural University 24: 205-209. [In Chinese with English summary]

Maynard Smith J. 1974. Models in ecology. Cambridge University Press, Cambridge: 1-35.

McCallum D. A. and Dixon P. M. 1990. Reducing bias in estimates of the Richards growth function shape parameter. Growth, Development and Aging 54: 135-141.

Parks J. R. 1982. A theory of feeding and growth of animals. Springer-Verlag, Berlin: 1-24.

Richards F. J. 1959. A flexible growth function for empirical use. Journal of Experimental Botany 10: 290-300.

Ricklefs R. E. 1968. Patterns of growth in birds. Ibis 110: 419-451.

Ricklefs R. E. 1976. Growth rates of birds in the humid new world tropics. Ibis 118: 179-207. 
Robertson T. B. 1908. On the normal rate of growth of an individual and its biochemical significance. Archiv für Entwicklungsmechanik der Organismem 25: 581-614.

Spillman W. J. and Lang E. 1924. The law of diminishing increment. Yonkers World, New York: 1-26.

STATISTICA for Windows Release 4.5 A. 1993. StatSoft Inc. Tulusa, Oklahoma, USA.

Verhulst P. F. 1838. Notice sur la loi que la population suit dans son accroissement. Correspondance Mathematique et Physique Publiee par A. Quetelet, Brussels 10: 113-121.

Wan X. R., Zhong W. Q. and Wang M. J. 1998a. New flexible growth equation and its application to the growth of small mammals. Growth, Development and Aging 62: 27-36.

Wan X. R., Zhong W. Q. and Wang M. J. 1998b. Ecology and management of rodent pest on the Brandt vole (Microtus brandti). [In: Ecology and management of rodent pests in agriculture. Z. B. Zhang and Z. W. Wang, eds]. Ocean Press, Beijing: 209-220. [In Chinese]

Zhou L., Liu J. K. and Liu Y. 1987. Studies on population productivity ecology of the plateau pika I. A dynamic model of growth of body weight in the plateau pika. Acta Theriologica Sinica 7: 67-87. [In Chinese with English summary]

Zhu B. and Xia J. 1991. Growth and development of brown bear under handrearing. Chinese Journal of Zoology 26: 37-39. [In Chinese with English summary]

Received 20 January 1999, accepted 6 October 1999. 